\title{
Les études hydrologiques sur la Haute-Durance
}

\section{Hydrological investigations on the upper Durance}

\author{
PAR I. SERRA,

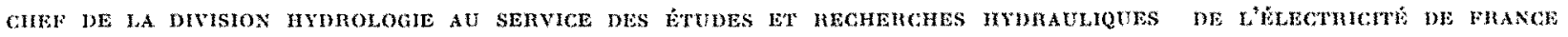

\begin{abstract}
Elude des facteurs conditionnels des débits : choix des cmplacements des postes plaviometriques en fonction des trois venls plavient principaux : vent du Midi, Lombarde et Marin. Mise an point de plnviomètres à angets basculeurs, munis d'enregistreurs le longue durke et chatufes atz propane. Mesure de la quantite de neige restant sur le sol par source radio-actione el compteur Geiger-Muller. Enregistrement des temperatures, du vent an sol. Etude géologique clu terrain.

Etude des débits a la station de l'Archidiacre: aménagement de la station, hydrogramme de crue, influence de la drtie de la pluie, de son átendue dans l'espace el de sa direction de propagation sur la forme de l'hydrogramme. Utilisation des probabilites d'apparition et de propagation des pluies.
\end{abstract}

En dépit du nombre relativement ćlevé de stations de jaugeage ayant fonctionne sur le bassin supérieur de la Durance, les débits de ce cours d'eau - débits moyens et surtoul débits extrémes (etiages et crues) - sont encore assez mal connus.

Les observations anciennes ef les courbes de larage de la plupart des stations sont trop souvent sujettes à caution. Souvent aussi des lacunes viennent fâcheusement interrompre les sérics de relevés. Tout cela rend difficile, sinon parfois impossible, l'utilisation des méthodes statistiques.

Nous avons donc été conduits à attaquer le problème d'une autre façon. Et au lieu de décrire sculement comment ont évolué les débits de la Durance, nous essaierons de comprendre pourquoi ils ont évolué dans tel ou tel sens en vue

\begin{abstract}
Study of the factors conditioning the discharge; choice of sites for plaviometrie pos/s, accomnt being taken of the three main rain winds: south wind, Lombardy wind and ocean wind. Development of tip-container plubiometers equipped with recording instruments and heatud by propane. Measurement of quantity of snow remaining on ground by radionactivily and Geiger-Muller counlers. Recording of lemperutures and of wind at ground-lewel. Geological stady of the land. Study of the discharges at the Archidiacre gatuging post : equipment of the post, flood hydrogramme, influence of length of rainfall period, of the surface it coners and of its direction of propagation on the shape of the hydrogramme. Itilization of probabilities of rain oceurence and propagation.
\end{abstract}

de dégager les lois qui régironl les deoulements futurs.

Une telle analyse du phenomine ne peut se faire que par l'étude ninuticuse, portant sur quelques années, des fincteur's essenticls qui conditionnent les débits.

Précisons immédiatement que le soin apporté a l'équipement de la Haule-Durance en appareils d'observations hydrométéorologiques se justific par l'importance des aménagements envisages. Le barrage de Serre-Ponçon, avec ses $100 \mathrm{~m}$ de hauteur, retiendra plus d'un milliard de $\mathrm{m}^{\prime \prime}$ d'eau. C'est dire l'intérêt qui s'attache à la comnaissance la plus exacte possible de la crue maximum pour pouvoir dimensionner les ouvrages évacuateurs et se prémunir ainsi contre les risques de catastrophes que la rupture d'un tel barrage ne manquerait pas de provoquer à l'aval. 


\section{I. - ÉTUDE DES FACTEURS CONDITIONNELS DU DÉBIT}

\section{A. - LES PRECIPITATIONS}

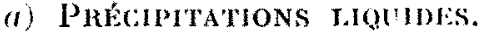

Au début de nos études, nous avons trouvé en place un réseau de postes pluviométriques, installés soil par la Météorologie Nationale, soit par le Centre Hydrométéorologique de Lyon (Direction de l'Exploitation E.D.F.).

Mais ce réseau nous est vite apparu insuffisant :

- A quelques rares exceptions près, les postes étaient situés dans des fonds de vallées. Donc peu ou pas de renseignements pluviométriques sur les régions de haute altitude et loin de tous lieux habités.

- Il n'était et il n'est encore fait dans ces postes qu'une seule lecture journalière. Or, les sources de la Durance ou de ses affluents ne se trouvent qu'à une centaine de kilomètres au plus de Serre-Ponçon el le flot met moins de vingt-quatre heures pour venir des points extrêmes.

Nous avions donc besoin de connaître, en plus de leur intensité totale, les heures de début et de fin d'averse ainsi que la forme des « diagrammes de pluies $\gg$.

C'est ainsi que nous avons été conduits à faire un large emploi des pluviomètres enregistreurs.

\section{Choix des emplacements des postes pluviométriques.}

La Haute-Durance (en englobant aussi sous ce terme les bassins versants du Guil et de l'Ubaye, c'est-à-dire en somme tout le bassin versant de Serre-Poncon) est soumise à l'influence de trois vents pluvieux d'origine, de nature et d'aire d'application différentes.

1. Le "Vent du Midi *, qui soulfle du S.-S.W. : on peut dire tres brievement que les pluies qui l'accompagnenl sont d'origine atlantique. Elles sont généralisées sur tout le bassin, mais avec plus ou moins d'intensité suivant les conditions locales d'orientation et dc relief.

Plus abondantes sur la Moyenne-Durance et le Guil que dans le bassin de l'Ubaye, elles sont beaucoup plus faihles encore sur la Durance Supérieure.

2. La « Lombarde », d'E.-S.E., vent dominant au voisinage de la frontière italienne, fréquemment associé à la pluie ou à la neige. Dans la région qui nous intéresse, son aire d'application se limite à la haute vallée de la Durance et à un degré moindre à celle du Guil. L'Ubaye est peu affectée.

3. Le « Marin » enfin, vent du S.E., qui amène des pluies d'origine méditerranéenne. Leur aire d'extension ne va pas au nord plus loin que le bassin de l'Ubaye. Elles n'intéressent donc la région ctudiée que pour une part assez faible.

Disons tout de suite - mais nous y reviendrons plus loin - qu'il ne peut y avoir coexistence de ces trois vents pluvieux. On n'aura donc pas à craindre l'arrive sinultanée à Serre-Ponçon de trois flots importants.

De même, il ne peut $y$ avoir en même temps superposition de pluies abondantes et de fonte rapide du manteau neigeux due à un effet du föehn. Le föehn souffle ici de l'E.-S.E. et ne peul coexister avee un vent de pluie généralisée du S.W.

\section{Installation des appareils.}

Sur la base de ce schéma de distribution des pluies dans le bassin de Serre-Ponçon, nous nous sommes done attachés à mettre en place un réseau d'appareils enregistreurs permettant de suivre, dans le temps et dans l'espace, l'évolution des perturbations pluvieuses.

Pas de difficultés spéciales pour l'installation des postes au voisinage des lieux habités. Nous avons utilisé les pluviomètres enregistreurs classiques à augets basculeurs, auxquels nous avons adjoint pour la saison froide un dispositif de chauffage électrique alimenté par le réseau.

Pour les régions éloignées ou d'accés difficile, nous avions envisagé plusieurs solutions :

1. Utilisation de nivompluviometres totalisateurs. Mais ces appareils présentent l'inconvínient grave de ne donner que le total des précipitations recueillies entre le moment de l'installation - en général octobre -.. et le moment où se font les relerés - fin de printemps et souvent octobre de l'année suivanle. On les a done abandonnés.

2. Utilisation de pluviomètres enregistreurs classiques munis de dispositifs de télémesure par fil ou sans fil. Solution abandonnée aussi en raison de trop grands risques de pannes d'exploitation en hiver.

3. Mise au point d'enregistreurs spéciaux de longue durée et spécialement adaptés aux conditions particulièrement sévires d'emploi en haute montagne. 
Type retenu : pluviomètre à augets basculeurs. - Enregistreur commandé par une horloge électrique alimentée par des piles spéciales résistant aux plus grands rroids. - Autonomie de marche : six mois.

Inscription sur papier paraffiné, par une pointe sèche pour éviter l'encrassement des plumes dû au gel de l'encre. Longueur du rouleau : $60 \mathrm{~m}$, avancement : $12,5 \mathrm{~mm}$ à l'heure par saccades toutes les secondes.

Chauffage: il ne pouvait être question de chauffage électrique (distances trop grandes pour «tirer » des lignes risquant d'ailleurs d'être rompues par le gel ou les ava- lanches). D'où système de chaufrage inedit par bouteilles de propane it $33 \mathrm{~kg}$ (8 bouteilles en série donnant une autonomie de six mois). - Brûleur double spécial commandé par thermostat.

L'ensemble des enregistreurs est contenu dans un cofrre mélallique calorifugé à double paroi (bourré de $5 \mathrm{~cm}$ de laine de verre). Le coflre est en cléments démontables pour le transporl à dos de mulets. Le tout est fixe sur une charpente métallique avec plateforme a $2 \mathrm{~m}$ de haut, le réceptacle du pluviometre se trouvant ainsi à $3,50 \mathrm{~m}$ au-dessus du sol.

La carte I indique les emplacements :

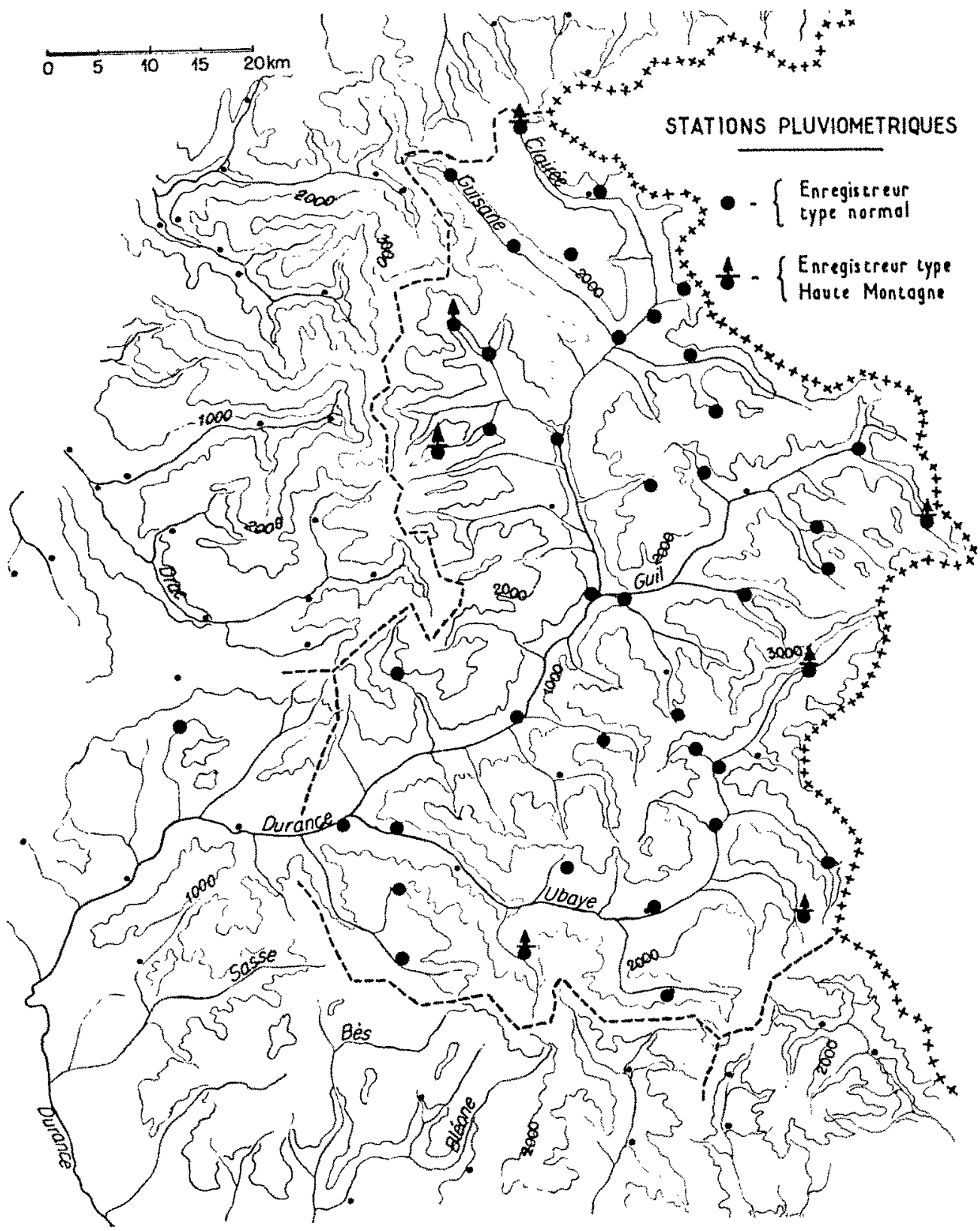

CAITE 1 


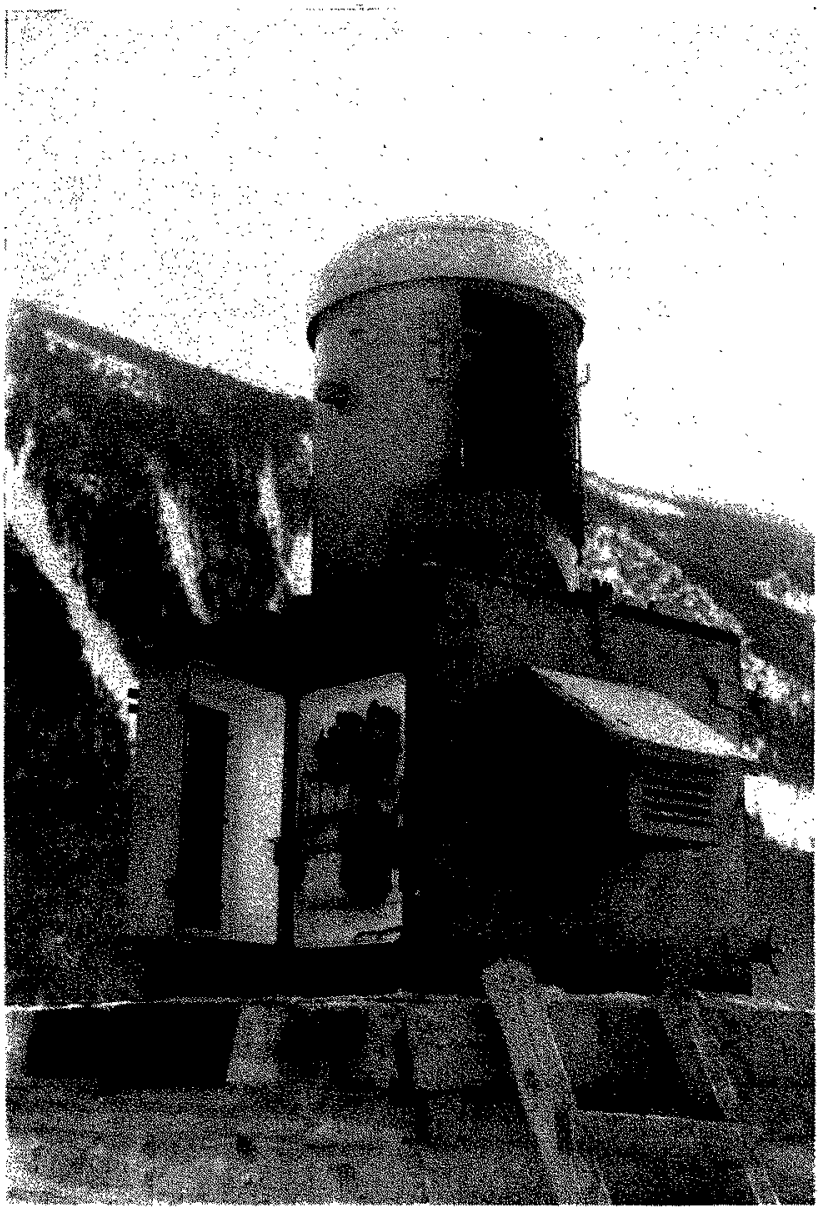

Puore 1. "Puviomele enregistreur de hate montagne. Vue d'ensemble (appareil ouvert);

a lextérieur, sur le pannea de droite. la niche du thermometre curesistrent.



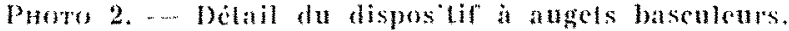
On distingue a la partie superieurc ha collerelte de chauflage de lentomoir.

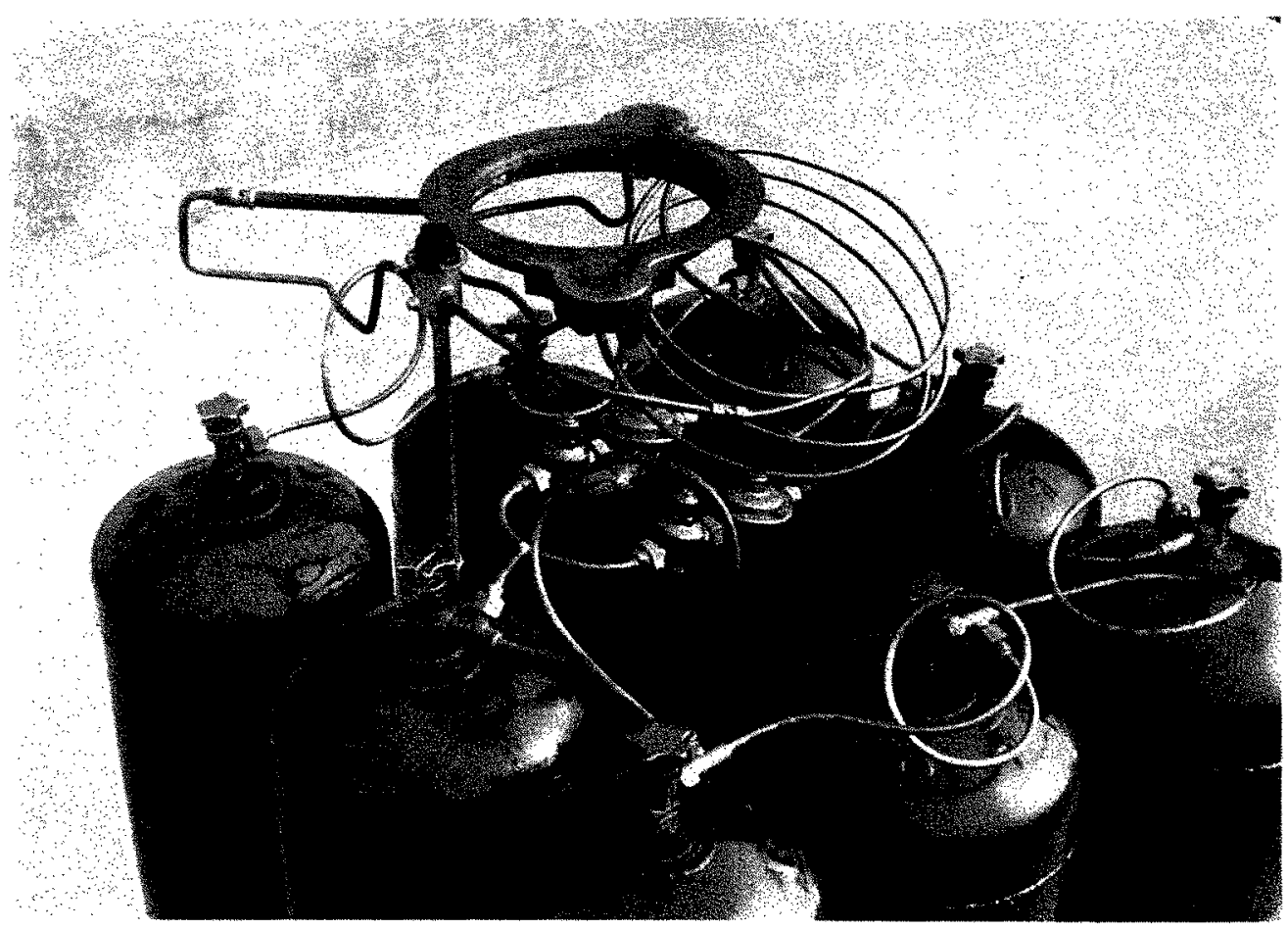

Puoro 3, D... Dispostif de thathase.

Les huit bouteiles de propane en serie avec leurs delenteurs, fo fhermostat ef la collerette de chauffage. 




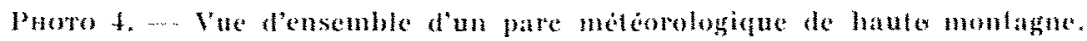

A droile du pluviometre cnestistrenr, les deux mâts-support

du comptenr de Geiger-Muller pour la mesure de lepaisseur de neige par radio-isotopes.

-. Des enregistreurs type courant 34 appareils en service);

Des enregistreurs type spécial de haute montagne $(10$ prévus dont 7 en service, tous à des altitudes comprises entre 2.000 et $2.400 \mathrm{~m}$ ). Quelques photos donnent des vues d'ensemble et de détail de ces appareils.

\section{b) Pricipitations solidfs.}

Deux genres de mesures sont effectués:

a) Mesure de la quantité de neige tombée.

Elle est faite d'une part par les pluviomètres enregistreurs eux-mêmes.

D'autre part, aupres des postes pluviométriques simples (où il n'est fait qu'une lecture journaliere) sont disposées soit des cuves à neige, soit des tables à neige à bascule donnant le total journalier de la précipitation tombée sous forme solide. b) Mesure de la quantité de neige restant sur le sol nécessaire pour étudier le mécanisme de fusion du stock neigeux ef son influence sur les debits).

Là aussi, deux méthodes :

-. Des perches à neige ont été installées sur le bassin. Au cours de tournées périodiques se font les lectures de la hauteur de neige. La densité (d'où l'on tire la \& valeur en eau $»)$ est déterminée par carrotlage d pesée. Mais les tournées sont rares parce que longues, difficiles et parfois dangereuses.

- Reprenant une idee trouvée dans des publications americaines, nous avons done mis at point un dispositif permettant d'aroir la valeur du stock neigeux de facon automatique el continue.

Le principe en est simple:

Si $I_{6}$ est lintensité d'un rayonnement ra- 
dio-actif, lorsqu'on interpose sur son trajet un écran d'épaisseur $x$, le rayonnement traversant l'écran a pour intensité :

$$
\mathrm{I}=\mathrm{I}_{0} e^{-\mu x}
$$

1. est une constante ne dependant que de la nature du matériau constituant l'écran. Elle est en particulier indépendante de létat physique de ce dernier. Dans le cas de la neige, le rapport $I / I_{0}$ ne dépend done que de la «hauteur d'eau équivalente» qui est justemen! l'élément qui nous intéresse.

Pratiquement, le dispositif comprend en gros :

- Une source radio-active ( $100 \mathrm{mc}$. de cohalt 60$)$ placée au niveau du sol et émettant à la verticale un pinceau fin de rayons $\gamma$.

- Un compteur de Gisger-Mularen maintenu à $4 \mathrm{~m}$ du sol dans le faisceau des rayons.

- Un enregistreur de « tops $\gg$ alimenté par une horloge électrique. - Bande de papier paraffiné se déroulant à vitesse constante.

Nous n'entrerons pas dans les détails techniques de fonctionnement. Notons seulement que l'appareil effectue 4 mesures par jour avec une autonomie de 8 mois.

Ces appareils de mesure de neige par radioisotopes sont placés près des pluviomètres enregistreurs de haute montagne dans des petits pares météorologiques clos de barbelés. (Voir leur emplacement carte I.)

\section{B. - LES TEMPERATURES}

- Dans les postes pluviométriques de vallée, il est fait deux lectures de température par jour.

- De plus, chacun des dix postes de haute montagne comporte sur la paroi extérieure du coffre un thermometre a tension de vapeur. Le distributeur d'impulsions du pluviomètre aclionne aussi le style d'un enregistreur thermo- métrique. On a done un enregistrement continu des températures qui se fait sur la mème bande de papier paraffiné que les relevés pluviométriques.

$$
\text { C. - LE VENT }
$$

- Vent en altitude (pour l'étude de la propagation des perturbations pluvieuses).

Nous utilisons pour cela les observations tres détaillées faites à la station d'Embrun qui est un «poste synoptique » du réseau de la Météorologie Nationale.

Des observations moins détaillées mais également utiles sont faites aussi au poste auxiliaire synoptique du Bois de l'Ours à Briançon.

- Vent au sol (pour l'étude des rafales au sol et leur infiuence sur les indications des pluviomètres - et pour l'étude de l'évaporation et la fusion du stock de neige).

Nous envisageons d'adjoindre à cinq des postes pluviométriques de haute montagne des appareils enregistrant la vilesse et la direction du vent. Ces anémomètres et girouettes seront placés sur des supports de $4 \mathrm{~m}$ de haul. Pour l'instant, nous en sommes à l'étude d'un prototype (en soufflerie, en chambre froide à - $40^{\circ}$ et dans la nature) pour voir surtout comment se comporteront les parties tournantes en cas de givre. Nous pensons pouvoir mettre les appareils en place dans le courant de l'été 1954

\section{c. - NATURE GEOIOGIQUE DES SOIS}

Notre programme comporte également l'blablissement d'une carte hydrogéologique de la Haute-Durance telle qu'elle a été dessinée pour certaines régions de la Tunisic. Nous sommes pour cela en relation avec les géologues qui ont inauguré et mis au point un tel mode de représentation des terrains, et a moins d'imprévu une campagne de prospection et d'études sur le terrain pourra être entreprise dès que les conditions atmosphériques le permettront.

\section{III. - ETUDE DES DEBITS}

Concurremment à l'étude des facteur's condilionncls du débit, nous poursuivons naturellement l'élude des débils eux-mêmes.

Un certain nombre de stations de jaugeage existaient déjà sur la Durance et ses afluents. Une étude critique des condilions dans lesquelles elles furent exploitées nous a d'abord permis d'extraire quelques résultats utilisables d'une masse informe de releves. Puis eerlaines de ces stations ont été "reprises » et re-équipées. 1)'autres stations nouvelles ont été enfin mises en service.

Ce sont en général des stations du type elassique munies de limnigraphes ef sur lesquelies i] 
est inutile d'insister. Leurs emplacements ont été reportés sur la carle II.

Les jaugeages se font par la méthode chimique (sauf en trois stations de la Durance ou le brassage n'est plus suffisant: ia lioche-de-Rame, Saint-Clément et l'Archidiacre).

Les courbes de larage viennent d'ètre terminées. Elles seront, bien entendu, tenues à jour.

Parmi toutes les stations de jaugeage du bassin, une mention spéciale doit ètre réservée à l'Archidiacre. C'est pour ainsi dire la * stalion principale $\gg d u$ bassin, la plus voisine de l'em- placement de Serre-Ponçon, donc celle où peuvent être mesurés à très peu de chose prós les débits qui alimenteront le barrage. (Le seul bassin versant intermediaire entre serre-poneon el l'Archiadiacre est en effet constilue par colui du petit torrent de Theus el celui de la Blanche qui au surplus sera plus tard dérivé dans SerrePonçon - soit $167 \mathrm{~km}^{2}$ sur un lotal de 3.884 , c'est-à-dire environ $4 \%$ ).

La station de l'Archidiacre a élé suffisamment décrite par ailleurs.

Rappelons seulement que les aminagements qui lui ont été apportés (passerelle mohile rou-

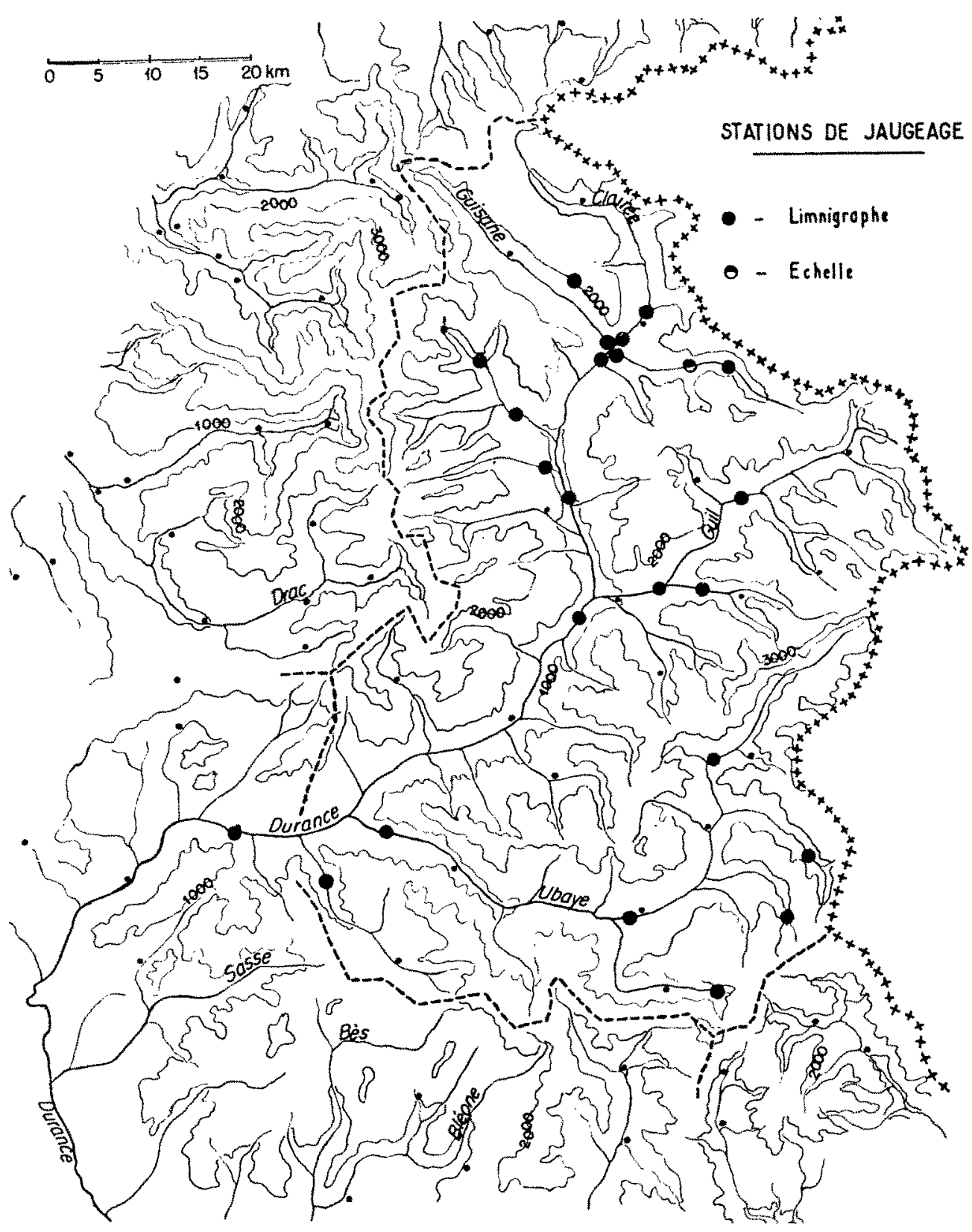

Cante 2 
lant sur rails) permettent des jaugeages au moulinet même par les plus fortes crues. Au surplus, un appareil à prélèvement continu permet de déterminer de facon précise el détaillée la courbe des débits solides en fonction du temps.

Enfin, le charriage des materiaux esí évalue par la formule de Mexen-P'rTen, la granulometric étant déterminée au moyen de prélèvements par nasses à graviers, ef l'appréciation du début de charriage faite par un détecteur hydrophonique dont l'enregistrement est efrectuć sur un magnétophone.

\section{III. - UTILISATION DES DONNÉES EXPÉRIMENTALES}

Chaque bassin versant élémentaire, avec sa slation de jaugeage et ses pluviomètres, constitue un «ensemble». La corrélation entre débits et pluies ou autres éléments méléorologiques permet de déterminer pour chacun d'eux l'hydrogramme correspondant à telle ou telle condition initiale.

Nous montrerons a titre d'exemple le genre d'études effectuées sur la station de l'Archidiacre, totalisatrice $d u$ bassin de Serre-Poncon.

Le problème essentiel est ici celui de la crue maximum à prévoir.

Une première «information » préalable et que l'on ne saurait négliger nous est donnée par la station de Ventavon un peu à l'aval $\left(4.216 \mathrm{~km}^{2}\right.$ au lieu de $3.834 \mathrm{~km}^{2}$ ) où trente années de mesure ont montré que les crues de la Durance dans ce secteur sont assez nettement localisées dans le temps.

Elles apparaissent :

- Soit en fin de printemps, c'est-à-dire en maijuin. La fonte des neiges participe au gonflement des débits;

..... Soit en automne: septembre-octobre-novenbre. Les crues sont alors dues aux précipitations seules.

Il doit donc a priori exister deux types d'hydrogrammes de crues.

.Jusqu'à présenl, l'unité de temps ne pouvait etre que le jour puisqu'on ne faisait aux stations pluviométriques qu'une lecture journalière. Nos appareils enregistreurs nous permettront de serrer davantage la réalite.

Sans entrer dans le détail de la méthode qui pourra faire l'objet d'un autre exposé, rappelons que :

a) Si une pluie couvre la totalite du bassin, la forme de l'hydrogramme de l'écoulement (c'està-dire débit en fonction du temps) dépend, pour une intensité de pluie donnée, d'une part de la durée de la pluie comparéc au temps de concentration a la station, d'autre part de la forme même du diagramme de pluie (toutes ces quanlités peuvent être mesurées: b) Si la pluie ne couvre qu'une partie du bassin, elle peut :

...- Soit traverser le bassin perpendiculairement á la direction générale du cours d'eau, el l'on a là un cas andogue à celuı de l'orage stationnaire sur une partic du bassin;

-. Soit ic traverser parallèlement au sens de l'écoulement. Si elle remonte le sens de l'ecoulement, on aura une suite de crues élémentaires donnant à la limite un hydrogramme aplati et allongé. Si elle descend au contraire le sens de l'écoulement, il conviendra de comparer sa vitesse de marche $V$ avec la vitesse $n$ de propagation de l'onde de crue. Le cas le plus défavorable est celui où $\mathrm{V}=v$.

L'hydrogramme devient alors tris pointu et l'on observera une montée des eaux rapide et intense.

Appliquons ces quelques considérations forcément très résumées au cas de Serre-Poncon.

L'orientation générale de la Durance est sensiblement N.E.-S.W. Nous avons vu, dans l'étude des vents pluvieux, que des pluies abondantes venant du N.E. et descendant tout le long de la Durance n'étaient pas à craindre. Donc, pas de crue intense avec montée d'eau très rapide du lype désigné plus haut sous le nom de "cas le plus défavorable ».

Mais nous pourrons avoir les cas suivants :

a) Pluies d'ouest (de N.W. à S.W.) courrant tout le bassin. Elles peuvent être continues, mais d'intensité relativement faible: hydrogramme aplati.

b) Pluies de S.E. plus intenses mais limities à la partie inférieure du hassin : hydrogramme pointu et crues plus dangereuses.

c) Succession de pluies de ces différents secteurs :

Si elles se font dans l'ordre: pluies de S.E. suivies de pluies d'W. (schéma $b+a$ ); on n'observera it Serre-Ponçon qu'une suite de crues dímentaires. 
Si elle se fait dans lordre inverse (schéma $a+b)$, on aura d'abord un débit soutenu et pouvant être relativement élevé dù aux pluies généralisées d'ouest. C'est sur ce flot de base que viendra so superposer lhydrogramme pointu dù aux pluies de S.E. An total, possibilité de forte crue.

C'est cette possibilité que nous nous nous eflorcons maintenant de chiffrer.

Nous sommes pour cela en relation itroile avec les Services de Climatologie et de Prévision de la Météorologie Nationale.

Un certain nombre d'études fort importantes ont déjà été effectućes sur l" Evolution des types de temps en Europe Occidentale ». Citons surtout l'actuelle mise à jour d'un calendrier, dit " calendrier de Barn », qui donne depuis I881 la suite chronologique de foules les silualions isobariques observeses au niveatu de la mer el classées en 26 types de lemps principaux.

partant de ces travaux de base, il doil alro possible de determiner pour une region lelle que lc. Sud-Est de la France:

Lal probabilité d'apparition de phénomenes successifs : en particulier pour le prom blème des crues de Serre-Poncon, pluies d'onest suivies de pluies de S.E.;

-... La probabilité de vilesse de marche de ces phénomènes, ou bien la probabilité de persistance des pluies exeeptionnellement abondantes et la quantité maximum à attendre en vingt-quatre heures, en douze heures ou en moins de temps encore;

Les prohabilités de réchauffement ou de baisse de temperature et leur corrcilation avec l'apparition de précipitations abondantes. Ces points sont particulièrement intéressants à examiner pendant les périodes - printemps et début de l'été _ au cours desquelles la fusion nivale participe nour une large part an gonflement des débits.

$$
\text { * }
$$

Nous avions dit au début de cette note que les mithodes stalistigues nous paraissatent demploi difteile sinon impossible pour létude des débils de la Durance. Nous y voici ecpendanl revenus mais par une roie détournée. C'est que le probleme n'est plas lo mème : il ne s'agil plus de traiter par lappareil mathemalique des siries plus on moins longues ef surtoul plus ou moins valables de relevés de débits el d'extrapoler les résultats jusquaux valeurs limiles qui sont les débits de crue.

Les observalions faites sur ha Hate-l)urame a l'aide de nos appareils enregistreturs doivent permettre d'analyser le phenomine de l'écoulement (e'est-à-dire le passage pluies-débits) et do déterminer ansi en ehague bassin partiel el pour le bassin total la forme des " hydrogrammes unilates s. El ce n'esl que ar stadc franchi, que la Statistique apparailrat : l'avaluation des probabilités dappartion de la on led lype de perlurbations pluvieuses permellat on definitive, of comple tenu des " informations expromentales $*$ recucillies sur le bassin puis fraruiles en hydrogrammes, de resoudre le probleme qui nows clail posé : la delermination de la crue maximum a craindre is Serre-Poneon.

$$
\text { * }
$$

Nous ne lorminerons pas ce rapide exposé sams souligner l'absolue nécessité pour l'élude d'un probleme de celle importance d'une stroite collaboration entre Services divers:

- - Service des Etudes at Rocherehes Hydrauliques, charge de la coordination of de l'blaboration des bludes.

- Région d'Equipement Hyolratioue Appes III rui a mis en place, parfois dams des eonditions tries dures, foutes les stations de mesures phuxiometrigues el hydrometriques ef en assure lexploitation.

-... Services de la Météorologie Nationale enlin, dont les budes sur les types de lempes et leurs possibilites d'apparition constituent un maillon indispensable dans l'itude qui a été entreprise.

\section{DISCUSSION}

(Président: M. Andwnwe?

M. He Président remercie M. Senna el somligne l'interét de l'étude synthétique de la Durance qui a fait l'objet de son exposé : il mppelle que le projet d'aménagement de cette rivière, necessitant des bases hydrologiques plus précises et complètes que eclles que lon pouvat lirev des statistiques disponibles, a motive celle etude physique des facteurs de lévolution des débits au moyen d'appareils de mesure particulierement consus a cet effet.
Sur la demande de M. Derrato, M. Skrka indique que Le coût unitaire mogen approximatif d'un appareil protolype utilise est de londre de 600.000 franes.

M. It President precise que ce prix rolativement deve est da pour une large part a la mise au point d'un appareillage nouveato dans une region difficile.

M. Dewart demante quel est finteret exict de len- 
registrement de la pluie et de la neige puisque celle-ch, notamment, ne fond pas continuellement à l'altitude du bussin.

M. Sekna précise que la mesure de la neige par radiom isotope n'est faile que quatre fois par jour, et que, d'autre part, l'enregistrement de la pluie qui tombe sert. it etudier le mécanisme de la fusion de la neige sous l'effet de la chute de la pluie.

M. le Président, appuye par M. Cnescext, confrme cet effet en rappelant qu'il a élé notamment à l'origine des crues du Haut-Drac en 1928 : eet effet n'est pas dù à la temperature des gouttes de pluie, mais à lair chaud qui accompagne généralement leur chute.

M. Senha ajoute que les thermometres enregistrenrs de l'appareillage décrit précisent cette temperature de l'air. Bien que moins intense que dans les Alpes septentriomales, l'effet de föhn est sensible dans le haut bassin di la Durance (Aont-Genève, col de l'Isoard).

II. le Président ayant rappelé la complexilé de l'exolution des debits, insiste sur l'intéret de l'utilisation des rayons $\gamma$ pour la pesée à distance des matières d'un mélange dans un solide donné : le procédé a de nombreuses applicatious ef semble tout à fait justifié pour mesurer périodiquement l'épaisseur de la neige en un point dacès diffeile. Un autre aspect à retenir de l'intérèt des rayons $\gamma$ pour l'expérimentation est laccumulation d'énergie sous un petit volume et une forme très maniable.

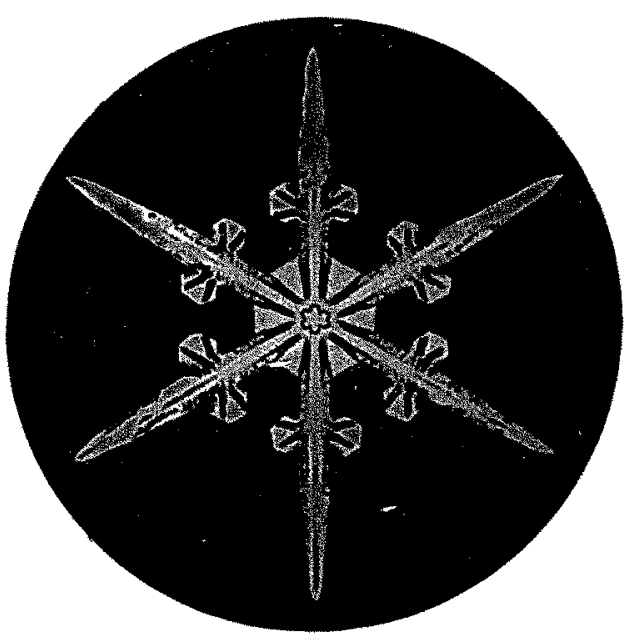

